\title{
SCATTERING THEORY AND POLYNOMIALS ORTHOGONAL ON THE REAL LINE
}

\author{
BY
}

\author{
J. S. GERONIMO AND K. M. CASE
}

\begin{abstract}
The techniques of scattering theory are used to study polynomials orthogonal on a segment of the real line. Instead of applying these techniques to the usual three-term recurrence formula, we derive a set of two two-term recurrence formulas satisfied by these polynomials. One of the advantages of these new recurrence formulas is that the Jost function is related, in the limit as $n \rightarrow \infty$, to the solution of one of the recurrence formulas with the boundary conditions given at $n=0$. In this paper we investigate the properties of the Jost function and the spectral function assuming the coefficients in the recurrence formulas converge at a particular rate.
\end{abstract}

I. Introduction. In our previous paper [7] the techniques of scattering theory were applied to polynomials orthogonal on the unit circle (POUC). The natural equations satisfied by these polynomials are two two-term recurrence formulas instead of the more familiar three-term recurrence formula satisfied by polynomials orthogonal on the real line (PORL). These two two-term recurrence formulas have several interesting properties. For example, the Jost function, which has been shown to be so useful in the theory of orthogonal polynomials [4], [7], is the limit of a sequence of polynomials satisfying one of the recurrence formulas with the boundary condition given at $n=0$. It is natural to ask whether such a system of recurrence relations exists for PORL.

In this paper we develop the theory of PORL along a line that parallels the theory of POUC and delve deeper into the consequences of applying scattering theory to PORL.

In §II we define the polynomials and derive the familiar three-term recurrence formula. Now, in analogy with POUC, a set of two two-term recurrence formulas is derived. These formulas plus the appropriate boundary conditions are taken as the fundamental equations defining the polynomials. From them the ChristoffelDarboux formula and Wronskian theorem are derived.

In §III the Jost function is defined and is shown to be the limit of a sequence of polynomials satisfying one of the recurrence formulas with the boundary conditions given at $n=0$. Some of the properties of the Jost function are investigated.

Since we have started with the recurrence relations it is necessary to show that the polynomials are indeed orthogonal. This is done in §IV. We also show how one can calculate the Jost function directly from the weight function.

Received by the editors August 9, 1978.

AMS (MOS) subject classifications (1970). Primary 42 A52.

(C) 1980 American Mathematical Society 0002-9947/80/0000-0159/\$08.00 
Next $(\S \mathrm{V})$ the discrete analogs of the Marchenko equations are derived [3]. The equations are then used to investigate the behavior of the Jost function at the points $z= \pm 1$. The consequences of these results for the weight function are discussed.

II. Preliminaries. Suppose we are given some nondecreasing $\rho(\lambda)$ [10] with infinitely many points of increase on a segment of $[a, b]$ of the real line such that

$$
s_{n}=\int_{a}^{b} \lambda^{n} d \rho(\lambda)
$$

exists for all $n$. We are to find polynomials $p(\lambda, n)$, such that

(i) $\rho(\lambda, n)$ is a polynomial of precise degree $n$ in which the coefficient of $\lambda^{n}$ is positive,

(ii) $\int_{a}^{b} p(\lambda, n) p(\lambda, m) d \rho(\lambda)=\delta(n, m), m, n=0,1,2, \ldots$

Using standard orthogonalization procedures one finds

$$
p(\lambda, n)=\left[H_{n-1} H_{n}\right]^{-1 / 2}\left|\begin{array}{ccccccc}
s_{0} & s_{1} & s_{2} & \cdot & \cdot & \cdot & s_{n} \\
s_{1} & s_{2} & s_{3} & \cdot & \cdot & \cdot & s_{n+1} \\
\cdot & \cdot & \cdot & \cdot & \cdot & \cdot & \cdot \\
s_{n-1} & s_{n} & \cdot & \cdot & \cdot & \cdot & s_{2 n-1} \\
1 & \lambda & & & & & \lambda^{n}
\end{array}\right|, \quad n>0
$$

where

$$
H_{n}=\left|\begin{array}{ccccccc}
s_{0} & s_{1} & s_{2} & \cdot & \cdot & \cdot & s_{n} \\
s_{1} & s_{2} & \cdot & \cdot & \cdot & \cdot & s_{n+1} \\
\cdot & \cdot & \cdot & \cdot & \cdot & \cdot & \cdot \\
s_{n} & s_{n+1} & \cdot & \cdot & \cdot & \cdot & s_{2 n}
\end{array}\right|, \quad n>0 .
$$

(Here we define $H_{-1} \equiv 1$ thus $p(\lambda, 0)=H_{0}^{1 / 2}=s_{0}^{-1 / 2}$.) The coefficient of $\lambda^{n}$ in $p(\lambda, n)$ can be determined from equation (II.2) and is ${ }^{1}$

$$
k(n)=\left(H_{n-1} / H_{n}\right)^{1 / 2}, \quad n=0,1, \ldots
$$

From the orthogonality condition one can construct the well-known three-term recurrence relation [10]

$$
\begin{aligned}
a(n+1) p(\lambda, n+1)+b(n) p(\lambda, n)+a(n) p(\lambda, n-1)= & \lambda p(\lambda, n), \\
& n=1,2, \ldots,
\end{aligned}
$$

where

$$
a(n)=k(n-1) / k(n)
$$

and

$$
b(n)=\int_{a}^{b} \lambda p(\lambda, n)^{2} d \rho(\lambda)
$$

Note that (II.j) can be extended to $n=0$ provided we define

$$
p(\lambda,-1)=0 \text {. }
$$

We shall also set

$$
a(0)=1 \text {. }
$$

\footnotetext{
1 The theory of positive quadratic forms tells us that the $H_{n}$ are positive (see [10D). Results were presented at the A.M.S. meeting \# 753 Columbus, Ohio, March 25, 1978.
} 
Thus we could take (II.5) and the initial conditions (II.8) and

$$
p(\lambda, 0)=k(0)
$$

as the starting point in our study of orthogonal polynomials. However one of our purposes is to develop the properties of polynomials orthogonal on the unit circle and those on a segment of the real line along similar lines. With this in mind consider the following two two-term recurrence formulas, ${ }^{2}$

$$
\begin{aligned}
p(\lambda, n)=\frac{a(\infty)}{a(n)}\left[(z-B(n-1)) p(\lambda, n-1)+\frac{1}{z} \psi(z, n-1)\right] & \\
& n=1,2, \ldots,
\end{aligned}
$$

and

$$
\begin{aligned}
& \psi(z, n)=\frac{a(\infty)}{a(n)}\left[\frac{1}{z} \psi(z, n-1)\right. \\
&+\left\{\left(1-\frac{a(n)^{2}}{a(\infty)^{2}}\right) z-B(n-1)\right\}p(\lambda, n-1)], \\
& n=1,2, \ldots,
\end{aligned}
$$

where we assume that

$$
\lim _{n \rightarrow \infty} a(n)=a(\infty)>0, \quad \lim _{n \rightarrow \infty} b(n)=b(\infty), \quad b(n) \text { real, }
$$

and we have set

$$
B(n)=\frac{b(n)-b(\infty)}{a(\infty)}
$$

and

$$
\lambda=a(\infty)(z+1 / z)+b(\infty) .
$$

By solving (II.11) for $\psi$ and then substituting the result into (II.12) one easily obtains (II.5).

Considering $p(\lambda, n)$ and $\psi(z, n)$ as two components of a function $\Phi$ defined by

$$
\Phi(z, n)=\left(\begin{array}{l}
p(\lambda, n) \\
\psi(z, n)
\end{array}\right),
$$

equations (II.11) and (II.12) can be condensed to

$$
\Phi(z, n)=C(n) \Phi(z, n-1)
$$

where

$$
C(n)=\frac{a(\infty)}{a(n)}\left[\begin{array}{lr}
z-B(n-1) & \frac{1}{z} \\
\left\{\left(1-\frac{a(n)^{2}}{a(\infty)^{2}}\right) z-B(n-1)\right\} & \frac{1}{z}
\end{array}\right] .
$$

\footnotetext{
${ }^{2} \psi(z, n)$ is a function of $z$ and $1 / z$.
} 
We will take the recurrence relations and the initial conditions

$$
p(\lambda, 0)=\psi(z, 0)=1 / \sqrt{s_{0}} \neq 0
$$

as the fundamental equations in our discussion of these orthogonal polynomials.

As a first application let us examine special cases of the following relation ${ }^{3}$

$$
\begin{aligned}
\Phi^{(1)}(z, n)\left[\begin{array}{cc}
0 & -1 \\
1 & 0
\end{array}\right] \Phi^{(2)}\left(z^{\prime}, n\right) \\
\quad=\Phi^{(1)}(z, n-1) C^{(1) T}(n)\left[\begin{array}{cc}
0 & -1 \\
1 & 0
\end{array}\right] C^{(2)}(n) \Phi^{(2)}\left(z^{\prime}, n-1\right),
\end{aligned}
$$

where $\Phi^{(1)}(z, n)$ and $\Phi^{(2)}\left(z^{\prime}, n\right)$ satisfy (II.16), (II.17) and (II.18) for $z$ and $z^{\prime}$, respectively. Substituting in (II.18) yields

$$
\begin{aligned}
= & \Phi^{(1)}(z, n-1) \frac{a(\infty)^{2}}{a(n)^{2}}\left[\begin{array}{ll}
z-B(n-1) & \left\{\left(1-\frac{a(n)^{2}}{a(\infty)^{2}}\right) z-B(n-1)\right\} \\
\frac{1}{z} & \frac{1}{z}
\end{array}\right] \\
& \cdot\left[\begin{array}{cc}
0 & -1 \\
1 & 0
\end{array}\right]\left[\begin{array}{ll}
z^{\prime}-B(n-1) & \frac{1}{z^{\prime}} \\
\left(1-\frac{a(n)^{2}}{a(\infty)^{2}}\right) z^{\prime}-B(n-1) & \frac{1}{z^{\prime}}
\end{array}\right] \Phi^{(2)}\left(z^{\prime}, n-1\right)
\end{aligned}
$$

which is

$$
=\Phi^{(1)}(z, n-1)\left[\begin{array}{ll}
B(n-1)\left(z-z^{\prime}\right) & -z / z^{\prime} \\
z^{\prime} / z & 0
\end{array}\right] \Phi^{(2)}\left(z^{\prime}, n-1\right) .
$$

Setting $z=z^{\prime}$ (Wronskian theorem) in (II.22) yields

$$
\begin{aligned}
& \Phi^{(1)}(z, n)\left[\begin{array}{cc}
0 & -1 \\
1 & 0
\end{array}\right] \Phi^{(2)}(z, n) \\
& \quad=\Phi^{(1)}(z, n-1)\left[\begin{array}{cc}
0 & -1 \\
1 & 0
\end{array}\right] \Phi^{(2)}(z, n-1) \equiv W\left[\Phi^{(1)}, \Phi^{(2)}\right] .
\end{aligned}
$$

Thus the Wronskian $W$ is independent of $n$.

With $\Phi^{(1)}=\Phi^{(2)}$ (Christoffel-Darboux), it can be shown [6] after some manipulation that

$$
\begin{aligned}
& a(n)\left(p(\lambda, n) p\left(\lambda^{\prime}, n-1\right)-p\left(\lambda^{\prime}, n\right) p(\lambda, n-1)\right) \\
& =\left(\lambda-\lambda^{\prime}\right) p\left(\lambda^{\prime}, n-1\right) p(\lambda, n-1) \\
& \quad+a(n-1)\left(p(\lambda, n-1) p\left(\lambda^{\prime}, n-2\right)-p(\lambda, n-2) p\left(\lambda^{\prime}, n-1\right)\right) .
\end{aligned}
$$

${ }^{3} C^{T}$ means transpose here. 
III. Jost function. Let us look at equation (II.18) for large $n$. Using (II.13) and (II.14), we have

$$
C^{0}=\lim _{n \rightarrow \infty} C(n)=\left[\begin{array}{ll}
z & 1 / z \\
0 & 1 / z
\end{array}\right] .
$$

Proceeding formally we introduce two auxiliary solutions

$$
\Phi_{+}(z, n)=\left(\begin{array}{l}
p_{+}(z, n) \\
\psi_{+}(z, n)
\end{array}\right)
$$

and

$$
\Phi_{-}(z, n)=\left(\begin{array}{l}
p_{-}(z, n) \\
\psi_{-}(z, n)
\end{array}\right)
$$

satisfying (II.17) and (II.18) with boundary conditions

$$
\begin{aligned}
\lim _{n \rightarrow \infty}\left|p_{ \pm}(z, n)-z^{ \pm n}\right|=0, & |z| \leq 1, \\
\lim _{n \rightarrow \infty}\left|\psi_{+}(z, n)\right|=0, & |z|<1,
\end{aligned}
$$

and

$$
\lim _{n \rightarrow \infty}\left|\psi_{-}(z, n)-\left(1-z^{2}\right) z^{-n}\right|=0, \quad|z|>1
$$

From (II.23)

$$
W\left[\Phi_{-}, \Phi_{+}\right]=-\left(z^{2}-1\right) .
$$

Therefore $\Phi_{+}(z, n)$ and $\Phi_{-}(z, n)$ are linearly independent except at $z= \pm 1$ and

$$
(z-1 / z) \Phi(z, n)=C_{+} \Phi_{+}(z, n)-C_{-} \Phi_{-}(z, n), \quad|z|=1 \text {, }
$$

where

$$
C_{ \pm}=W\left[\Phi, \Phi_{ \pm}\right]
$$

which is

$$
C_{+}=p_{+}(z, n) \psi(z, n)-p(\lambda, n) \psi_{+}(z, n)
$$

and

$$
C_{-}=p_{-}(z, n) \psi(z, n)-p(\lambda, n) \psi_{-}(z, n)
$$

Since (III.8) and (III.9) are independent of $n$ we can evaluate them for large $n$. Thus

$$
C_{+}=\lim _{n \rightarrow \infty} z^{n} \psi(z, n)=\lim _{n \rightarrow \infty} \psi^{*}(z, n)
$$

where

$$
\psi^{*}(z, n)=z^{n} \psi(z, n)
$$

and

$$
C_{-}(z)=\lim _{n \rightarrow \infty}\left(z^{-n} \psi(z, n)-\left(1-z^{2}\right) z^{-n} p(\lambda, n)\right) .
$$

Letting $z \rightarrow 1 / z$ in (II.11) and subtracting it from the original equation gives us

$$
\left(1-z^{2}\right)(1 / z) p(\lambda, n-1)=(1 / z) \psi(z, n-1)-z \psi(1 / z, n-1) .
$$


Substituting this result into (III.12) yields

$$
C_{-}(z)=\lim _{n \rightarrow \infty} z^{2} z^{-n} \psi(1 / z, n) .
$$

Now define

$$
f_{+}(z)=\frac{a(\infty)}{k(0) z} C_{+}(z)=\frac{a(\infty)}{k(0) z} W\left[\Phi, \Phi_{+}\right]
$$

and

$$
f_{-}(z)=\frac{a(\infty)}{k(0) z} C_{-}(z)=\frac{a(\infty)}{k(0) z} W\left[\Phi, \Phi_{-}\right]
$$

Equation (III.6) now becomes

$$
(z-1 / z) \Phi(z, n)=\frac{k(0)}{a(\infty)}\left[f_{-}(z) \Phi_{+}(z, n)-f_{+}(z) \Phi_{-}(z, n)\right], . \quad|z|=1 .
$$

Using (III.10) and (III.11) it is easy to see that ${ }^{4}$

$$
\overline{f_{+}(z)}=f_{+}(1 / z)=f_{-}(z)
$$

for $|z|=1$.

If one uses (II.11) to eliminate $\psi(z, n)$ and $\psi_{+}(z, n)$ in (III.8), one finds ${ }^{5}$

$$
f_{+}(z)=\frac{a(n+1)}{k(0)}\left[p(\lambda, n+1) p_{+}(z, n)-p_{+}(z, n+1) p(\lambda, n)\right]
$$

or

$$
f_{+}(z)=p_{+}(z,-1)
$$

We shall call $f_{+}(z)$ the Jost function for polynomials orthogonal on a segment of the real line.

In order to investigate the properties of the Jost function, we will find it convenient at this point to introduce the techniques of Banach algebras. Thus, let $A$ denote the class of functions integrable on $-\pi<\theta<\pi$ such that if $g$ is an element of $A$ then

$$
g(\theta) \approx \sum_{k=-\infty}^{\infty} g(k) e^{i k \theta}
$$

with

$$
\|g\|=\sum_{k=-\infty}^{\infty}|g(k)|<\infty .
$$

Let $A^{+}$and $A^{-}$denote those functions in $A$ of the form

$$
g(\theta) \approx \sum_{k=0}^{\infty} g(k) e^{i k \theta}
$$

4 Note from (II.11) and (II.12) that the coefficients of $f_{+}(z)$ are real.

5 This corresponds to Case's definition, see [2]. 
and

$$
h(\theta) \approx \sum_{k=-\infty}^{0} h(k) e^{i k \theta}
$$

respectively.

Let $\|g\|$ be the norm on $A, A^{+}$and $A^{-}$, then $A, A^{+}$and $A^{-}$are Banach algebras [3].

THEOREM 1. If

$$
\sum_{n=1}^{\infty} n\left\{\left|1-\frac{a(n)^{2}}{a(\infty)^{2}}\right|+|B(n-1)|\right\}<\infty
$$

then

(i) ${ }^{6} z f_{+}(z)$ is analytic inside and continuous on the unit circle,

(ii) $z f_{+}(z)$ is an element of $A^{+}$,

(iii) if $f_{+}(z)$ has zeros inside the unit circle they are
(a) real,
(b) simple,
(c) finite in number,
(d) the points where the orthogonal polynomials are square summable.

Finally

(iv) if $f_{+}(z)$ has zeros on the unit circle

(a) they must be at $z=+1$ and $/$ or $z=-1$,

(b) they must be simple.

Property (iii) is proved in Appendix A. Properties (i) and (ii) are proved in Appendix B. Property (iv, b) is proved in $\S V$. Property (iv, a) is a consequence of (III.17) and (III.18) and the fact that the zeros of $p(\lambda, n)$ alternate with those of $p(\lambda, n-1)[4]$.

In Appendix B the following is also proved:

Theorem 2. If (III.25) holds then $p_{+}(z, n)$ is analytic inside the unit circle, continuous on it, and is an element of $A^{+}$.

LEMMA 1. $p_{-}(z, n)$ is analytic outside the unit circle, continuous on it, and is an element of $A^{-}$.

From the boundary conditions and recurrence formulas one finds that

$$
p_{+}(z, n)=p_{-}(1 / z, n) \text {. }
$$

The above formula and Theorem 2 prove the lemma.

LEMMA 2. If $f_{+}(z)=0$ at $z=1$, then

$$
z f_{+}(z)=(1-z)^{\alpha} g(z)
$$

where $\alpha<1$ and $g(z)$ is bounded at $z=1$, continuous everywhere else on the unit circle, and analytic for $|z|<1$.

\footnotetext{
6 Properties (i), (iii) and (iv) have been independently proved by Guseinov, see [8]. Property (i) has also been proved by Nevai, see [9].
} 
Proof. The lemma follows from Theorem 1(i), Theorem 2, Lemma 1 and (III.17).

Throughout the rest of this paper we will assume that the coefficients in the recurrence formulas satisfy (II.13) and (III.25).

IV. Consequences. Having defined the Jost function and listed some of its properties in the prevous section, we now investigate the role it plays in various properties of orthogonal polynomials.

THEOREM 3 (ORTHOGONALITY CONDITION). If (II.13) and (III.25) hold then

$$
\int_{-\infty}^{\infty} p(\lambda, n) p(\lambda, m) d \rho(\lambda)=\delta(n, m)
$$

where

$$
d \rho(\lambda) \approx \begin{cases}\sigma(\lambda) d \lambda, & b(\infty)-2 a(\infty)<\lambda<b(\infty)+2 a(\infty), \\ \sum_{i=1}^{N} \rho_{i} \delta\left(\lambda-\lambda_{i}\right) d \lambda, & \lambda \text { not as above, } \lambda_{i} \neq \lambda_{j} \text { for } i \neq j, N<\infty\end{cases}
$$

with

$$
\sigma(\lambda) d \lambda=\frac{a(\infty) \sin \theta d \lambda}{\pi k(0)^{2}\left|f_{+}\right|^{2}}, \quad \lambda=a(\infty)(z+1 / z)+b(\infty), z=e^{i \theta},
$$

and

$$
\rho_{i}=\frac{p_{+}\left(z_{i}, 0\right)}{k(0)^{2} f_{+}^{\prime}\left(\lambda_{i}\right)}, \quad \lambda=a(\infty)\left(z_{i}+1 / z_{i}\right)+b(\infty),\left|z_{i}\right|<1 .
$$

Here $\lambda_{i}$ denote the roots of $f_{+}(z)$ for $|z|<1$.

THEOREM 4 (RELATION TO WEIGHT FUNCTION). If (II.13) and (III.25) hold then

$$
\begin{aligned}
& f_{+}(z)= \frac{d(z) \pi_{+}\left(z_{i}-z\right) \pi_{-}\left(z-z_{i}\right)}{z \Pi_{i=1}^{n}\left(1-z z_{i}\right)} \\
& \cdot \exp \left\{-\frac{\left(z-z^{-1}\right)}{4 \pi} \int_{b(\infty)-2 a(\infty)}^{b(\infty)+2 a(\infty)} \frac{d \lambda^{\prime} \ln }{\sin \theta^{\prime}\left(\lambda^{\prime}-\lambda\right)}\left(\frac{\pi k(0)^{2}\left|d\left(z^{\prime}\right)\right|^{2} \sigma\left(\lambda^{\prime}\right)}{a(\infty) \sin \theta^{\prime}}\right)\right\}, \\
& \lambda=a(\sigma)\left(z^{\prime}+1 / z^{\prime}\right)+b(\infty), z^{\prime}=e^{i \theta^{\prime}},|z|<1 .
\end{aligned}
$$

Here $\left\{z_{i}\right\}$ are the roots of $f_{+}(z)$ for $|z|<1$ and $\pi_{ \pm}$means the product of $z_{i}$ in the unit circle subject to $z_{i} \lessgtr 0$. $d(z)$ is equal to

(1) 1 if $f_{+}(z) \neq 0$ for $z= \pm 1$,

(2) $1-z$ if $f_{+}(z)=0$ only at $z=1$, for $z=e^{i \theta}$,

(3) $1+z$ if $f_{+}(z)=0$ only at $z=-1$, for $z=e^{i \theta}$,

(4) $1-z^{2}$ if $f_{+}(z)=0$ at $z= \pm 1$.

To prove Theorem 3 examine the following integral

$$
I=\frac{1}{2 \pi i} \oint_{z^{-1}} \frac{p_{+}(z, n) p(\lambda, m)}{C_{+}(z)} d z, \quad m<n,|z|=1 .
$$


The existence of the integral is a consequence of Theorem 1(i) and (iv, a), Theorem 2 , and Lemma 2. Solving (III.17) for $p_{+}(z, n)$ then substituting this and (III.15) into the above equation yields

$$
\begin{aligned}
\frac{1}{2 \pi i} \oint_{z^{-2}} \frac{p_{+}(z, n) p(\lambda, m)}{(k(0) / a(\infty)) f_{+}(z)} d z & \\
= & \frac{1}{2 \pi i} \oint \frac{z^{-2} p(\lambda, m) p(\lambda, n)}{\left(k(0)^{2} / a(\infty)^{2}\right)\left|f_{+}(z)\right|^{2}}(z-1 / z) d z \\
& +\frac{1}{2 \pi i} \oint \frac{z^{-1} p_{-}(z, n) p(\lambda, m)}{(k(0) / a(\infty)) z f_{-}(z)} d z, \quad|z|=1, n>m .
\end{aligned}
$$

Letting $\theta \rightarrow-\theta$ in the second term on the right-hand side and combining it with the left-hand side gives

$$
\begin{aligned}
& \frac{1}{2 \pi i} \oint \frac{z^{-2} p(\lambda, n) p(\lambda, m)(z-1 / z)}{\left(k(0)^{2} / a(\infty)^{2}\right)\left|f_{+}(z)\right|^{2}} d z \\
& \quad=\frac{-1}{2 \pi i} \oint \frac{p_{+}(z, n) p(\lambda, m)}{(k(0) / a(\infty)) f_{+}(z)}\left(1-1 / z^{2}\right) d z, \quad|z|=1, n>m,
\end{aligned}
$$

where we have used (III.18) and the fact that $p_{+}(z, n)=p_{-}(1 / z, n)$ for $|z|=1$. Now changing over to real variables in the integral on the left-hand side of the above equation

$$
=\frac{1}{2 \pi} \int_{-\pi}^{\pi} \frac{p(\lambda, m) p(\lambda, n) 2 i \sin \theta e^{-i \theta} d \theta}{\left(k(0)^{2} / a(\infty)^{2}\right)\left|f_{+}(z)\right|^{2}} \quad z=e^{i \theta}
$$

Breaking up the range of integration to $\int_{0}^{\pi}$ and $\int_{-\pi}^{0}$ letting $\theta \rightarrow-\theta$ in the second integral then recombining

$$
=\frac{1}{\pi} \int_{0}^{\pi} \frac{2 p(\lambda, m) p(\lambda, n) \sin ^{2} \theta d \theta}{\left(k(0)^{2} / a(\infty)^{2}\right)\left|f_{+}(z)\right|^{2}} \quad z=e^{i \theta} .
$$

Finally using (II.15), equation (IV.9) becomes

$$
=\int_{b(\infty)-2 a(\infty)}^{b(\infty)+2 a(\infty)} p(\lambda, m) p(\lambda, n)\left(\frac{a(\infty) \sin \theta}{\pi k(0)^{2}\left|f_{+}(z)\right|^{2}}\right) d \lambda .
$$

In order to continue we need the following limits for $z=0$.

$$
\begin{aligned}
& \text { (a) } f_{+}(z)=z^{-1} a(\infty) \prod_{i=1}^{\infty} \frac{a(\infty)}{a(i)}, \\
& \text { (b) } p(\lambda, n)=z^{-n} k(0) \prod_{i=1}^{n} \frac{a(\infty)}{a(i)} \\
& \text { (c) } p_{+}(z, n)=z^{n} \prod_{i=n+1}^{\infty} \frac{a(\infty)}{a(i)}
\end{aligned}
$$

These limits are easily obtained from the recurrence relations and boundary conditions satisfied by the functions above (see Appendix B). 
Returning to (IV.7) we evaluate the integral on the right-hand side using the above limits, Theorem 1(iii) and the residue theorem. Thus

$$
\begin{aligned}
-\frac{1}{2 \pi i} \oint \frac{p_{+}(z, n) p(\lambda, m)\left(1-1 / z^{2}\right)}{(k(0) / a(\infty)) f_{+}(z)} d z \\
=\delta(n, m)-\sum_{i=1}^{N} \frac{p_{+}\left(z_{i}, n\right) p(\lambda, m)}{(k(0) / a(\infty)) f_{+}^{\prime}\left(z_{i}\right)}\left(1-1 / z_{i}^{2}\right)
\end{aligned}
$$

where $\left\{z_{i}\right\}$ refers to the zeros of $f_{+}(z)$ for $|z|<1$. From Appendix A

$$
p_{+}\left(\lambda_{i}, n\right)=\frac{p_{+}\left(\lambda_{i}, 0\right)}{k(0)} p\left(\lambda_{i}, n\right)
$$

at a zero of $f_{+}(z)$ for $|z|<1$. Substituting this equation into (IV.12) and using the fact that

$$
\frac{f_{+}^{\prime}\left(z_{i}\right)}{a(\infty)\left(1-1 / z_{i}^{2}\right)}=f_{+}^{\prime}\left(\lambda_{i}\right)
$$

( refers to differentiation with respect to the variable of which $f_{+}$is a function) yields

$$
=\delta(n, m)-\sum_{i}^{N} p\left(\lambda_{i}, n\right) p\left(\lambda_{i}, m\right) \frac{p_{+}\left(\lambda_{i}, 0\right)}{k(0)^{2} f_{+}^{\prime}\left(\lambda_{i}\right)}, \quad n>m
$$

or

$$
\begin{aligned}
& \int_{b(\infty)-2 a(\infty)}^{b(\infty)+2 a(\infty)} p(\lambda, n) p(\lambda, m) \frac{a(\infty) \sin \theta}{\pi k(0)^{2}\left|f_{+}(z)\right|^{2}} d \lambda \\
& \quad+\sum_{i}^{N} p\left(\lambda_{i}, n\right) p\left(\lambda_{i}, m\right) \frac{p_{+}\left(\lambda_{i}, 0\right)}{k(0)^{2} f_{+}^{\prime}\left(\lambda_{i}\right)}=\delta(n, m), \quad n>m .
\end{aligned}
$$

For $m>n$ examine the integral

$$
I=\frac{1}{2 \pi i} \oint_{z^{-1}} \frac{p_{+}(z, m) p(\lambda, n)}{C_{+}(z)} d z .
$$

The result is the same as (IV.15) giving us the orthogonality relation. With the above relations the spectral function can be identified with the Jost function in the following way:

$$
\begin{aligned}
d \rho(\lambda) & \approx \sigma(\lambda) d \lambda, \quad 2 a(\infty)-b(\infty)<\lambda<2 a(\infty)+b(\infty) \\
& \approx \sum_{i}^{N} \rho_{i} \delta\left(\lambda-\lambda_{i}\right) d \lambda, \quad \lambda \text { not as above, } \lambda_{i} \neq \lambda_{j} \text { for } i \neq j, N<\infty
\end{aligned}
$$

where $\sigma(\lambda)$ and $\rho_{i}$ are given in (IV.2) and (IV.3).

However, usually the weight function is given and one must determine the Jost function. This can be done using the Poisson integral formula. For $f_{+}(z) \neq 0$ at 
$z= \pm 1$ the proof follows essentially that given in [4]. If $f_{+}(z)$ has a zero on the unit circle then $f_{+}(z)$ must be modified in a way exhibited in $\S V .2$. The proof given in [4] may then be applied to $f_{+}(z) / d(z)$ yielding (IV.4).

V. Inverse scattering theory. We now introduce the techniques of inverse scattering theory. In particular the discrete analogs of the Marchenko equations are derived. These equations are then used to prove property $1(\mathrm{iv}, \mathrm{b})$ of §III. The consequences for the weight function of these results is discussed. In this section we assume that properties 1(i), (ii), (iii) and (iv, a) have been proved.

1. Derivation of the Marchenko equations. We are given a comparison system of orthogonal polynomials $\left\{p^{\prime}(\lambda, n)\right\}$ with

$$
a(n)=a(\infty)
$$

and

$$
B(n)=0
$$

for all $n$. From the recurrence formulas $p_{+}^{\prime}(z, n)=z^{n}$ and $p^{\prime}(\lambda, n)$ are Tchebycheff polynomials of the second kind. From Theorem 2 and Lemma 1 (see Appendix B)

$$
p_{+}(z, n)=\sum_{i=n}^{\infty} A(n, i) z^{i}
$$

and therefore

$$
p_{-}(z, n)=\sum_{i=n}^{\infty} A(n, i) z^{-i}
$$

where

$$
\sum_{i=n}^{\infty}|A(n, i)|<\infty
$$

Dividing (III.17) by $f_{+}(z)$ yields

$$
\left(z-\frac{1}{z}\right) \frac{p(\lambda, n)}{f_{+}(z)}=\frac{k(0)}{a(\infty)}\left[-p_{-}(z, n)+S(z) p_{+}(z, n)\right], \quad|z|=1,
$$

where

$$
S(z)=\frac{f_{-}(z)}{f_{+}(z)}=\frac{\overline{f_{+}(z)}}{f_{+}(z)}, \quad|z|=1 .
$$

Substituting (V.3) and (V.4) into (V.6) then multiplying by $(a(\infty) / k(0))\left(z^{m} / 2 \pi i\right)$ with $m>n$ and integrating around the unit circle yields

$$
\begin{aligned}
\frac{1}{2 \pi i} \oint \frac{p(\lambda, n)}{f_{+}(z)} z^{m} & \frac{a(\infty)}{k(0)}(z-1 / z) \frac{d z}{z} \\
= & -\sum_{n^{\prime}=n}^{\infty} A\left(n, n^{\prime}\right) \frac{1}{2 \pi i} \oint_{z^{m-n^{\prime}} \frac{d z}{z}} \\
& +\sum_{n^{\prime}=n}^{\infty} A\left(n, n^{\prime}\right) \frac{1}{2 \pi i} \oint S(z) z^{n^{\prime}+m} \frac{d z}{z}, \quad m>n>-1 .
\end{aligned}
$$


From (II.8) the L.H.S. is equal to zero when $n=-1$. Otherwise, we can evaluate the L.H.S. using the residue theorem and (IV.13), (IV.14), (IV.3) and Theorems 1(i) and 1(iii). Thus for $m>n \geqslant 0$

$$
\frac{1}{2 \pi i} \oint \frac{p(\lambda, n)}{f_{+}(z)} z^{m} \frac{a(\infty)}{k(0)}\left(z-\frac{1}{z}\right) \frac{d z}{z}=\sum_{i=1}^{N} \rho_{i} p_{+}\left(z_{i}, n\right) z_{i}^{m}, \quad m>n>0 .
$$

$\rho_{i}$ is defined in (IV.3). For $m=n$ there is a residue at zero which is equal to $-1 / A(n, n)$ [3], [5]. Substituting (V.3) and (V.9) and combining it with (V.8) gives the discrete analogs of the Marchenko equations

$$
\alpha(n, m)+w(n, m)+\sum_{n^{\prime}=n+1}^{\infty} \alpha\left(n, n^{\prime}\right) w\left(n^{\prime}, m\right)=0, \quad m>n>0,
$$

and

$$
1 / A(n, n)^{2}=1+w(n, n)+\sum_{n^{\prime}=n+1}^{\infty} \alpha\left(n, n^{\prime}\right) w\left(n^{\prime}, n\right), \quad m=n>0,
$$

where

$$
\alpha(n, m)=A(n, m) / A(n, n)
$$

and

$$
w(n, m)=-\frac{1}{2 \pi i} \oint S(z) z^{n+m} \frac{d z}{z}+\sum_{i=1}^{N} \rho_{i} z_{i}^{n+m} .
$$

For $n=-1$ we have

$$
\alpha(-1, m)+w^{\prime}(-1, m)+\sum_{n^{\prime}=0}^{\infty} \alpha\left(-1, n^{\prime}\right) w^{\prime}\left(n^{\prime}, m\right)=0, \quad m>-1,
$$

where $\alpha(-1, m)$ is defined as above and

$$
w^{\prime}(n, m)=-\frac{1}{2 \pi i} \oint S(z) z^{n+m} \frac{d z}{z} .
$$

From (V.7)

$$
|S(z)|=1 \text {. }
$$

The manipulations leading to (V.10), (V.11) and (V.14) can be justified using (V.15), (V.16) and the fact that $p_{+}(z, m)$ is an element of $A^{+}$.

The $\{a(i)\}$ in the recurrence relation are related to $\{A(i, i)\}$ in the following way $[5],[6]^{7}:$

$$
\frac{a(n)}{a(\infty)}=\frac{A(n, n)}{A(n-1, n-1)}, \quad n>0
$$

2. The properties of $f_{+}(z)$ at $z= \pm 1$. Equation (III.17) shows us that if $f_{+}(z)=0$ at $z=1$ then

$$
f_{+}(z)=(1-z)^{\alpha} g(z)
$$

7 This can be easily seen using (V.3) and (IV.11). 
where $\alpha<1$ and $g(z)$ is bounded at $z=1$. This is also true if $f_{+}(z)=0$ at $z=-1$. We show here that $\alpha=1$ and $g( \pm 1) \neq 0 .{ }^{8}$ Summing (V.14) from $m=n$ to $\infty$ gives $^{9}$

$$
\overline{\alpha(n)}+\sum_{m=n-1}^{\infty} \omega^{\prime}(m)+\sum_{n^{\prime}=0}^{\infty} \alpha\left(-1, n^{\prime}\right) \sum_{m=n^{\prime}+n}^{\infty} \omega^{\prime}(m)=0,{ }^{10}
$$

where

$$
\overline{\alpha(n)}=\sum_{m=n}^{\infty} \alpha(-1, m) .
$$

Summing by parts gives

$$
\begin{aligned}
0= & \overline{\alpha(n)}+\sum_{m=n-1}^{\infty} \omega^{\prime}(m)+\sum_{n^{\prime}=0}^{\infty} \alpha\left(-1, n^{\prime}\right) \sum_{m=n-1}^{\infty} \omega^{\prime}(m) \\
& +\sum_{n^{\prime}=0}^{\infty} \overline{\alpha\left(n^{\prime}\right)}\left(\sum_{m=n^{\prime}+n}^{\infty} \omega^{\prime}(m)-\sum_{m=n^{\prime}+n-1}^{\infty} \omega^{\prime}(m)\right)
\end{aligned}
$$

which is

$$
=\overline{\alpha(n)}+(1+\overline{\alpha(0)}) \sum_{m=n+1}^{\infty} \omega^{\prime}(m)-\sum_{n^{\prime}=0}^{\infty} \alpha\left(\overline{n^{\prime}}\right) \omega^{\prime}\left(n^{\prime}+n-1\right) .
$$

Assuming that $f_{+}(z)=0$ at $z=1$, equations (II.9), (III.20), (V.3), (V.12) and (V.17) say that

$$
1+\overline{\alpha(0)}=\frac{a(0) p_{+}(1,-1)}{a(\infty) A(0,0)}=\frac{f_{+}(1)}{a(\infty) A(0,0)}=0
$$

Therefore

$$
\overline{\alpha(n)}-\sum_{n^{\prime}=0}^{\infty} \alpha \overline{\left(n^{\prime}\right)} \omega^{\prime}\left(n^{\prime}+n-1\right)=0
$$

From (B.54)

$$
\sum\left|\omega^{\prime}(n)\right|<\infty \text {. }
$$

Therefore there exists $\overline{\omega(n)}, \hat{\omega}(n)$ and $N$ such that

$$
\begin{gathered}
\overline{\omega(n)}=\omega^{\prime}(n)-\hat{\omega}(n), \quad \hat{\omega}(n), \quad \overline{\omega(n)} \neq 0, \quad n<N, \\
\overline{\omega(n)}=\omega^{\prime}(n), \quad n>N,
\end{gathered}
$$

and

$$
\sum_{n^{\prime}=-1}^{\infty}\left|\overline{\omega\left(n^{\prime}\right)}\right|<1
$$

Substituting these results into (V.24) gives

$$
\overline{\alpha(n)}-\sum_{n^{\prime}=0}^{\infty} \overline{\alpha\left(n^{\prime}\right) \omega\left(n^{\prime}+n-1\right)}+\sum_{n^{\prime}=0}^{\infty} \overline{\alpha\left(n^{\prime}\right)} \hat{\omega}\left(n^{\prime}+n-1\right)=0
$$

\footnotetext{
8 This proof is adopted from the book of Agranovich and Marchenko, see [1].

9 All operations are justified in Appendix B.

${ }^{10}$ Note that from (V.15) $\omega^{\prime}(n, m)=\omega^{\prime}(n+m)$.
} 
Summing from $n=0$ to $\infty$ yields

$$
\begin{aligned}
\sum_{n=0}^{\infty} \overline{\alpha(n)}-\sum_{n=0}^{\infty} & \overline{\omega\left(n^{\prime}+n-1\right)} \sum_{n^{\prime}=0}^{\infty} \overline{\alpha\left(n^{\prime}\right)} \\
= & -\sum_{n=0}^{\infty} \hat{\omega}\left(n^{\prime}+n-1\right) \sum_{n^{\prime}=0}^{N+2} \overline{\alpha(n)}
\end{aligned}
$$

or

$$
\sum_{n=0}^{\infty}|\overline{\alpha(n)}|\left(1-\sum_{n^{\prime}=-1}^{\infty}\left|\overline{\omega\left(n^{\prime}\right)}\right|\right)<\sum_{n=0}^{N}|\hat{\omega}(n)| \sum_{n^{\prime}=0}^{N+2}\left|\overline{\alpha\left(n^{\prime}\right)}\right|
$$

Thus

$$
\sum_{n=0}^{\infty}|\overline{\alpha(n)}|<\frac{\sum_{n=0}^{N}|\hat{\omega}(n)| \sum_{n^{\prime}-0}^{N+2}\left|\overline{\alpha\left(n^{\prime}\right)}\right|}{1-\sum_{n^{\prime}=-1}^{\infty}\left|\overline{\omega\left(n^{\prime}\right)}\right|}<\infty
$$

Defining

$$
k(z)=\sum_{n=0}^{\infty} \overline{\alpha(n)} z^{n}
$$

equation (V.30) tells us that $k(z)$ is an element $A^{+}$and $k(z)$ is continuous at $z=1$. For $z \neq 1$ integrating (V.31) by parts gives

$$
k(z)=\frac{\overline{\alpha(0)}}{1-z}+\sum_{n=1}^{\infty} \sum_{j=n}^{\infty} z^{j}(\overline{\alpha(n)}-\overline{\alpha(n-1)})
$$

which

$$
=\frac{\overline{\alpha(0)}}{1-z}+\sum_{n=1}^{\infty} \frac{z^{n}}{1-z}(\overline{\alpha(n)}-\overline{\alpha(n-1)}) .
$$

Using (V.20) gives

$$
k(z)=\frac{1}{1-z}\left(1+\overline{\alpha(0)}-1-\sum_{n=1}^{\infty} z^{n} \alpha(-1, n-1)\right)
$$

or using (V.23), (III.20), (V.3) and (V.12)

$$
=\frac{-z}{1-z}\left(1 / z+\sum_{n=0}^{\infty} z^{n} \alpha(-1, n)\right)=\frac{-z}{1-z} \frac{a(0) p_{+}(z,-1)}{a(\infty) A(0,0)} .
$$

Thus

$$
=\frac{-z}{1-z} \frac{f_{+}(z)}{a(\infty) A(0,0)}
$$

Therefore $z f_{+}(z) /(1-z)$ is an element of $A^{+}$.

If $f_{+}(-1)=0$ then applying $\sum_{m=n}^{\infty}(-1)^{m}$ to (V.19), defining

$$
\overline{\alpha(n)}=\sum_{m=n}^{\infty}(-1)^{m} \alpha(-1, m)
$$

and using the previous techniques shows that $z f_{+}(z) /(1+z)$ is an element of $A^{+}$. 
Finally if $f_{+}(z)=0$ at $z= \pm 1$ then the previous results show that $z f_{+}(z) /(1-$ $z)$ and $z f_{+}(z) /(1+z)$ are elements of $A^{+}$. One now uses the local Weiner-Levy theorem [10] to show that $z f_{+}(z) /\left(1-z^{2}\right)$ is an element of $A^{+}$. Thus $z f_{+}(z) / d(z)$, $z=e^{i \theta}$, is an element of $A^{+} \cdot d(z)$ is defined following (IV.4). This proves property (iv, b). $\left(\overline{\left.z f_{+}(z) / d(z)\right)}\right.$ is an element of $A^{-}$, therefore $\left|z f_{+}(z) / d(z)\right|^{2}$ is an element of $A$. Since $\left|f_{+}(z) / d(z)\right|^{2} \neq 0$ for $|z|=1$ an application of the Wiener-Levy theorem tells us that

$$
\ln \left|f_{+}(z) / d(z)\right|^{2}
$$

is an element of $A$. Equation (IV.2) now implies the following:

THEOREM 5. If equations (II.13) and (III.25) hold then

$$
\ln \left(\frac{\pi k(0)^{2}|d(z)|^{2} \sigma(\lambda)}{a(\infty) \sin \theta}\right)
$$

is an element of $A$.

VI. Conclusion. In this paper the techniques of scattering theory have been used to study polynomials orthogonal on a segment of the real line. Since one of our aims was to try to parallel the theory of polynomials orthogonal on the unit circle, a set of two two-term occurrence formulas satisfied by polynomials orthogonal on a segment of the real line was derived. One of the advantages of these two two-term recurrence formulas is that the Jost function is just the limit of a sequence of polynomials satisfying one of the recurrence formulas with the boundary condition given at $n=0$. This, of course, simplifies the discussion of the analytic properties of the Jost function. In this paper we have assumed that a particular sum of the coefficients in the recurrence converges. The consequences of this assumption on the Jost function and weight function were discussed.

Appendix A. In this appendix some of the properties of the zeros of $\psi^{*}(z, n)$ and $f_{+}(z)$ are discussed. First it is shown that for $|z|<1$ the zeros of $\psi^{*}(z, n)$ are real, simple, and alternate with those of $p(\lambda, n-1)$. Equations (III.10) and (III.15) then imply that the zeros of $f_{+}(z)$ are real and simple ${ }^{11}$ for $|z|<1$. It is also shown that $p(\lambda, n)$ are square summable at a zero of $f_{+}(z)$ for $|z|<1$. Finally a proof is given showing that if the coefficients in the recurrence formula satisfy (III.25) there are only a finite number of points where $f_{+}(z)=0$ for $|z|<1$.

I. Zeros of $\psi^{*}(z, n)$. We begin our study with the following formula:

$$
\begin{aligned}
\frac{a(n)}{a(\infty)}\left[p^{1}(\lambda, n) p^{2}\left(\lambda^{\prime}, n-1\right)-p^{2}\left(\lambda^{\prime}, n\right) p^{1}(\lambda, n-1)\right] \\
=\left(z+1 / z-z^{\prime}-1 / z^{\prime}\right) \sum_{i=0}^{n-1} p^{2}\left(\lambda^{\prime}, i\right) p^{1}(\lambda, i) \\
\cdot \frac{a(0)}{a(\infty)}\left[p^{1}(\lambda, 0) p^{2}\left(\lambda^{\prime},-1\right)-p^{1}(\lambda,-1) p^{2}\left(\lambda^{\prime}, 0\right)\right]
\end{aligned}
$$

${ }^{11}$ This is true under conditions weaker than (III.25); see Appendix B. 
This equation can be derived from (II.5) [4] or (II.22) [6]. Solving for $(a(\infty) / a(n))(z$ $-B(n-1)) p(\lambda, n-1)$ in (II.11) and then substituting the result into (II.12) yields

$$
p(\lambda, n)-\psi(z, n)=\frac{a(n)}{a(\infty)} z p(\lambda, n-1) .
$$

Since $p^{1}(\lambda, i)$ and $p^{2}(\lambda, i)$ satisfy (II.11) they also satisfy (A.2). Therefore substituting (A.2) into the L.H.S. of (A.1) yields

$$
\begin{aligned}
p^{1}(\lambda, n) \psi^{2}\left(z^{\prime}, n\right)-p^{2}\left(\lambda^{\prime}, n\right) \psi^{1}(z, n) & \\
= & \left(z+1 / z-z^{\prime}-1 / z^{\prime}\right) \sum_{i=0}^{n-1} p^{1}\left(\lambda^{\prime}, i\right) p^{2}\left(\lambda^{\prime}, i\right) \\
& +\frac{a(0)}{a(\infty)}\left[p^{1}(\lambda, 0) p^{2}\left(\lambda^{\prime},-1\right)-p^{2}\left(\lambda^{\prime}, 0\right) p^{1}(\lambda,-1)\right] .
\end{aligned}
$$

Setting $p^{1}=\bar{p}=\bar{p}^{2}, \psi^{2}=\psi=\bar{\psi}^{1}$ and $z^{\prime}=\bar{z}$ yields

$$
\begin{aligned}
& (1 / z) \overline{p(\lambda, n)} \psi(z, n)-(1 / \bar{z}) p(\lambda, n) \overline{\psi(z, n)} \\
& \quad=(z+1 / z-\bar{z}-1 / \bar{z}) \sum_{i=0}^{n-1}|p(\lambda, i)|^{2}+(1 / z-1 / \bar{z})|p(\lambda, n)|^{2} .
\end{aligned}
$$

Thus the zeros of $\psi(z, n)$ for $|z|<1$ are real.

Now setting $p^{2}=p^{1}=p, \psi^{2}=\psi^{1}=\psi$ in (A.3) and letting $z^{\prime} \rightarrow z$ gives

$$
\begin{aligned}
((1 / z) \psi(z, n))^{\prime} p(\lambda, n)-(1 / z) \psi(z, n) p^{\prime}(\lambda, n) & \\
= & -(1 / z)^{2} p(\lambda, n)^{2}+\left(1-1 / z^{2}\right) \sum_{i=0}^{n-1} p(\lambda, i)^{2} .
\end{aligned}
$$

From the above equation we see immediately that the zeros of $\psi(z, n)$ are simple. Using an argument similar to that given by Szegö [10] proving that the zeros of $p(\lambda, n)$ and $p(\lambda, n-1)$ alternate, we can show that [6] the zeros of $\psi(z, n)$ and $p(\lambda, n-1)$ alternate for $|z|<1$. Also if $z_{i}$ is the smallest positive zero of $p(\lambda, n)$, $\psi(z, n)$ will have a zero at $z_{0}$ where $0<z_{0}<z_{i}$. Likewise let $z_{j}$ be the negative zero of $p(\lambda, n)$ with smallest magnitude then $\psi(z, n)$ will have a negative zero $z_{1}$ where $0<\left|z_{1}\right|<\left|z_{j}\right|$. Now using (III.10), (III.15), (B.12) and (B.17) we see that the zeros of $f_{+}(z)$ for $|z|<1$ are real and simple.

Setting $a(n+1)=a(\infty)$ and $B(n)=0$ for $n>n_{0}$ in (II.11) and (II.12) gives

$$
p^{n_{0}}(\lambda, n+1)=z p^{n_{0}}(\lambda, n)+(1 / z) \psi^{n_{0}}(z, n), \quad n>n_{0} .
$$

and

$$
\psi^{n_{0}}(z, n+1)=(1 / z) \psi^{n_{0}}(z, n), \quad n>n_{0}
$$

or

$$
\psi^{* n_{0}}(z, n+1)=\psi^{* n_{0}}(z, n)=\psi^{*}\left(z, n_{0}\right), \quad n>n_{0} .
$$

It is easy to see from (II.11) that

$$
p^{n_{0}}(\lambda, n)=p(\lambda, n), \quad n<n_{0},
$$

and

$$
\psi^{n_{0}}(z, n)=\psi(z, n), \quad n<n_{0} .
$$


From (III.10), (III.15) and (A.27)

$$
f_{+}\left(z, n_{0}\right)=\frac{a(\infty)}{k(0) z} \psi^{*}\left(z, n_{0}\right)
$$

where $f_{+}\left(z, n_{0}\right)$ is the Jost function for the system whose coefficients reach their asymptotic value for $n \geqslant n_{0}$. Substituting these results into (III.17) yields

$$
\begin{aligned}
\Phi^{n_{0}}(z, n)=\frac{k(0)}{a(\infty)(z-1 / z)}\left[f_{-}\left(z, n_{0}\right) \Phi_{+}^{n_{0}}(z, n)-f_{+}\left(z, n_{0}\right) \Phi_{-}^{n_{0}}(z, n)\right], & \\
& |z|=1 .
\end{aligned}
$$

In particular

$$
p^{n_{0}}(\lambda, n)=\frac{k(0)}{a(\infty)(z-1 / z)}\left[f_{-}\left(z, n_{0}\right) p_{+}^{n_{0}}(z, n)-f_{+}\left(z, n_{0}\right) p_{-}^{n_{0}}(z, n)\right]
$$

From (A.6) it is easy to see that

$$
p^{n_{0}}(\lambda, m)=z^{m-n_{p}}\left(\lambda, n_{0}\right)+z^{m-n_{0}-2} \psi\left(z, n_{0}\right) \sum_{i=0}^{m-n_{0}-1} z^{-2 i}, \quad m>n_{0} .
$$

Therefore as $m \rightarrow \infty p^{n_{0}}(\lambda, m)$ is unbounded for $|z|<1$ except at a zero $\left(z_{0}\right)$ of $\psi\left(z, n_{0}\right)$. In this case for $|z|<1$,

$$
p^{n_{0}}\left(\lambda_{0}, m\right)=z_{0}^{m-n_{0}} p\left(\lambda_{0}, n_{0}\right)
$$

Since $f_{-}\left(z_{0}, n_{0}\right)$ is a polynomial in $1 / z$ it can be continued inside the unit circle. Thus at a zero of $f_{+}\left(z, n_{0}\right)$ for $|z|<1$,

$$
p^{n_{0}}\left(\lambda_{0}, n\right)=\frac{k(0)}{a(\infty)\left(z_{0}-1 / z_{0}\right)} f_{-}\left(z_{0}, n_{0}\right) p_{+}^{n_{0}}\left(z_{0}, n\right), \quad\left|z_{0}\right|<1,
$$

or

$$
p^{n_{0}}\left(\lambda_{0}, n\right)=C^{n_{0}} p_{+}^{n_{0}}\left(z_{0}, n\right)
$$

where

$$
C^{n_{0}}=\frac{p\left(\lambda_{0}, 0\right)}{p_{+}^{n_{0}}\left(z_{0}, 0\right)}=\frac{k(0)}{p_{+}^{n_{0}}\left(z_{0}, 0\right)}
$$

At a zero of $\psi\left(z, n_{0}\right)$ equation (A.5) tells us that

$$
\begin{aligned}
\left(1 / z_{0}\right) \psi^{\prime n_{0}} & \left(z_{0}, n\right) p^{n_{0}}\left(\lambda_{0}, n\right) \\
& =-\left(1 / z_{0}^{2}\right) p^{n_{0}}\left(\lambda_{0}, n\right)^{2}+\left(1-1 / z^{2}\right) \sum_{i=0}^{n-1} p^{n_{0}}\left(\lambda_{0}, i\right)^{2}, \quad n>n_{0} .
\end{aligned}
$$

Substituting in the previous results gives

$$
\frac{f_{+}^{\prime}\left(z_{0}, n_{0}\right) k(0)^{2}}{a(\infty) p_{+}^{n_{0}}\left(z_{0}, 0\right)}=-\left(1 / z^{2}\right) p^{n_{0}}\left(\lambda_{0}, n\right)^{2}+\left(1-1 / z_{0}^{2}\right) \sum_{i=0}^{n-1} p^{n_{0}}\left(\lambda_{0}, i\right)^{2}
$$


Letting $n \rightarrow \infty$, using (A.13) and changing the differentiation of $f_{+}$from $z$ to $\lambda$ yields

$$
\frac{k(0)^{2} f_{+}^{\prime}\left(\lambda_{0}, n_{0}\right)}{p_{+}^{n_{0}}\left(z_{0}, 0\right)}=\sum_{i=0}^{\infty} p^{n_{0}}\left(\lambda_{0}, i\right)^{2} .
$$

If we now let $n_{0} \rightarrow \infty$ and use (III.10), (III.15) and (B.17) we see that at a zero $z_{i}$, $\left(\lambda_{i}\right)$ of $f_{+}(z)$ where $\left|z_{i}\right|<1$,

$$
p\left(\lambda_{i}, n\right)=\frac{k(0)}{p_{+}\left(z_{i}, 0\right)} p_{+}\left(z_{i}, n\right)
$$

and

$$
\frac{1}{\rho_{i}}=\frac{k(0)^{2} f_{+}^{\prime}\left(\lambda_{i}\right)}{p_{+}\left(z_{i}, 0\right)}=\sum_{j=0}^{\infty} p^{2}\left(\lambda_{i}, j\right)<\infty .
$$

Here $\rho_{i}$ is the same as in (IV.3).

II. The number of zeros of $f_{+}(z)$ for $|z|<1$. We now prove ${ }^{12}$ that if the coefficients satisfy (III.25) then $f_{+}(z)$ will have only a finite number of zeros inside the unit circle. Here we will find it convenient to use a version of (II.5)

$$
a^{\prime}(n+1) p\left(\lambda^{\prime}, n+1\right)+B^{\prime}(n) p\left(\lambda^{\prime}, n\right)+a^{\prime}(n) p\left(\lambda^{\prime}, n-1\right)=\lambda^{\prime} p\left(\lambda^{\prime}, n\right)
$$

where

$$
a^{\prime}(n)=a(n) / 2 a(\infty), \quad \lambda^{\prime}=\frac{1}{2}(z+1 / z)
$$

and

$$
B^{\prime}(n)=B(n) / 2
$$

Thus the continuous spectrum occurs for $|\lambda|<1$ and the eigenvalues (points where the polynomials are square summable) occur for $|\lambda|>1$.

The boundary value problem (A.22) and (II.8) is equivalent to finding solutions $y=\{y\}_{-1}^{\infty}$ to the equation

$$
J(y)=\lambda^{\prime} y
$$

with the boundary condition $y(-1)=0$. This in turn is equivalent to finding solutions $y=\{y\}_{0}^{\infty}$ to the equation

$$
\begin{aligned}
B^{\prime}(0) y(0)+a^{\prime}(1) y(1) & =\lambda^{\prime} y(0), \\
a^{\prime}(n) y(n-1)+B^{\prime}(n) y(n)+a^{\prime}(n+1) y(n+1) & =\lambda^{\prime} y(n), \quad n=1,2,3 \ldots
\end{aligned}
$$

Let $J^{\prime}$ denote the minimal closed operator induced by (A.25) in $l^{2}(0, \infty)$. It follows from (II.13) that $J^{\prime}$ is selfadjoint [2]. The domain of $J^{\prime}, D_{J^{\prime}}$, consist of all functions $y \in l^{2}(0, \infty)$ satisfying the following conditions:

(1) $y(n+1)$ and $y(n-1)$ must exist for all finite $n>1$,

(2) $J^{\prime}(y) \in l^{2}(0, \infty)$.

12 This proof has been adapted from the book of Agranovich and Marchenko (see [1D. 
We must show that the equation

$$
J^{\prime} y=\lambda^{\prime} y
$$

has only a finite number of eigenvalues.

To this end we introduce two additional selfadjoint operators $J_{1 m}$ and $J_{2 m}$ for $m>0$ defined in $l^{2}(0, m)$ and $l^{2}(m+1, \infty)$.

Where the equation for $J_{1 m}$ is

$$
\begin{aligned}
B^{\prime}(0) y(0)+a^{\prime}(1) y(1) & =\lambda^{\prime} y(0), \\
a^{\prime}(n) y(n-1)+B^{\prime}(n) y(n)+a^{\prime}(n+1) y(n+1) & =\lambda^{\prime} y(n), \\
n & =1, \ldots, m-2, \\
a^{\prime}(m-1) y(n-2)+B^{\prime}(m-1) y(n-1) & =\lambda^{\prime} y(m-1)
\end{aligned}
$$

and the equation for $J_{2 m}$ is

$$
\begin{aligned}
B^{\prime}(m+1) y(m+1)+a^{\prime}(m+2) y(m+2) & =\lambda y(m+1), \\
a^{\prime}(n) y(n-1)+B^{\prime}(n) y(n)+a^{\prime}(n+1) y(n+1) & =\lambda^{\prime} y(n), \\
n & =m+2, m+3, \ldots
\end{aligned}
$$

The domains $D_{J_{1 m}}$ and $D_{J_{2 m}}$ consist of functions satisfying the following conditions. For $y \in D_{J_{1 m}}$,

(1) $J_{1 m}(y) \in l^{2}(0, m)$,

(2) $y(n+1)$ and $y(n-1)$ exist for $1<n<m-1$.

For $y \in D_{J_{2 m}}$,

(1) $J_{2 m}(y) \in l^{2}(m+1, \infty)$

(2) $y(n+1)$ and $y(n-1)$ exist for all finite $n, n>m+2$.

Equations (A.27) and (A.28) are equivalent to equation (A.24) with the boundary conditions $y(-1)=y(m)=0$, and $y(m)=0$ respectively.

We now show that $J_{2 m}$ has no eigenvalues for large enough $m$. First let us note that since (A.25) is equivalent to (A.24) with the boundary condition $y(-1)=0$. We know from Appendix A that the eigenvalues of $J$ and therefore $J^{\prime}$ occur at the zeros of $f_{+}(z)$ for $|z|<1$. Thus the equivalence of $J_{2 m}$, and $J$ with the boundary condition $y(m)=0$, allows us to associate the eigenvalues of $J_{2 m}$ with the zeros of Jost function $f_{+}^{m-1}(z)$ where

$$
f_{1}^{m-1}(z)=a^{\prime}(m) p_{+}(z, m-1)
$$

for $0<|z|<1, z$ real.

From (B.37) and (B.41)

$$
\begin{aligned}
\mid p_{+}(z, m)-z^{m} & \prod_{i=m+1}^{\infty} \frac{1}{2 a^{\prime}(i)} \mid \\
<C^{\prime}|z|^{m+1} & \sum_{i=m+1}^{\infty} i\left\{\left|1-2 a^{\prime}(n+1)^{2}\right|+\left|2 B^{\prime}(n)\right|\right\} .
\end{aligned}
$$

Consequently for large enough $m=m_{0}, p_{+}\left(z, m_{0}\right)$ has no zeros for $0<|z|<1$ and $J_{2 m}$ has no point spectrum. 
We now show that $J_{1 m_{0}}$ has only a finite number of eigenvalues. Now $J_{1 m_{0}}$ is equivalent to $J^{\prime}$ with boundary condition $y\left(m_{0}\right)=0$. Since $J_{1 m_{0}}$ is a finite dimensional Jacobi matrix, it is easy to see that the eigenvalues of $J_{1 m_{0}}$ occur at the zeros of $p\left(\lambda^{\prime}, m_{0}\right)$. Since $p\left(\lambda^{\prime}, m_{0}\right)$ is a polynomial it has only a finite number of zeros. Let $k$ be the number of zeros of $p\left(\lambda^{\prime}, m_{0}\right)$ with $\left|\lambda^{\prime}\right|>1(|z|<1)$. It follows that the number, $N$, of eigenvalues of $J^{\prime}$ with $\left|\lambda^{\prime}\right|>1$ (number of zeros of $f_{+}(z)$ with $|z|<1)$ is

$$
N<k+1 \text {. }
$$

To prove this let us assume that $N>k+1$ and let

$$
g^{1}(n), g^{2}(n), \ldots, g^{k+2}(n)
$$

denote the $k+2$ linearly independent eigenvectors of $J^{\prime}$ corresponding to $k+2$ different eigenvalues whose magnitudes are greater than one. ${ }^{13}$

Turning our attention to $J^{\prime 2}, J_{1 m_{0}}^{2}$ and $J_{2 m_{0}}^{2}$ and their respective domains $D_{J^{\prime 2}}$, $D_{J_{1 m}^{2}}$ we note that from (A.26) $g^{i}(n)$ are also linearly independent eigenvectors of $J^{\prime 2}$. Now combine the eigenvectors to form $k+1$ linearly independent combinations $x^{1}(n), x^{2}(n), \ldots, x^{k+1}(n)$ such that

$$
x^{i}\left(m_{0}\right)=0, \quad i<k+1 \text {. }
$$

Consequently the $x^{i}$ belong to both $D_{J_{1 m_{0}}^{2}}$ and $D_{J_{2 m_{0}}^{2}}$. Since the zeros of $p\left(\lambda^{\prime}, m_{0}\right)$ are simple it is possible to form a nontrivial linear combination of the $x^{i}(n)$,

$$
f(n)=\sum_{i=1}^{k+1} z_{i} x^{i}(n)
$$

such that $f(n)$ is orthogonal to all the eigenvectors of $J_{1 m_{0}}^{2}$ corresponding to eigenvalues with magnitude greater than one. Since the $x^{i}(n)$ are in $D_{J_{1 m_{0}}^{2}}$ we can use the orthogonality condition on that space to impose $k$ conditions on $k+1$ unknowns. Denoting the scalar product $\Sigma_{\beta}^{\alpha} f(n) g^{*}(n)$ in $l^{2}(\alpha, \beta)$ as $\langle f, g\rangle(\alpha, \beta)$, the vector $f(n)$ satisfies the following relations:

$$
\left\langle J_{1 m_{0}}^{2} f f\right\rangle_{\left(0, m_{0}-1\right)}-\langle f f\rangle_{\left(0, m_{0}-1\right)}=\left\langle\left(J_{1 m_{0}}^{2}-1\right) f f\right\rangle_{\left(0, m_{0}-1\right)}<0
$$

since $f(n)$ is orthogonal to all the eigenvectors of $J_{1 m_{0}}^{2}$ with eigenvalues greater than 1 , and

$$
\left\langle J_{2 m_{0}}^{2} f f\right\rangle_{\left(m_{0}+1, \infty\right)}-\langle f f\rangle_{\left(m_{0}+1, \infty\right)}=\left\langle\left(J_{2 m_{0}}^{2}-1\right) f f\right\rangle_{\left(m_{0}+1, \infty\right)}<0
$$

since $J_{2 m_{0}}^{2}$ has no eigenvectors corresponding to discrete eigenvalues. But

$$
\left.\left\langle J^{\prime 2} f f\right\rangle_{(0, \infty)}-\langle f f\rangle_{(0, \infty)}=\left\langle\left(J^{\prime 2}-1\right) f f\right\rangle_{(0, \infty)}\right\rangle 0
$$

because $f(n)$ is just a linear combination of the eigenvectors of $J^{\prime 2}$ with eigenvalues greater than 1. But

$$
\left\langle\left(J^{\prime 2}-1\right) f f\right\rangle_{(0, \infty)}=\left\langle\left(J_{1 m_{0}}^{2}-1\right) f f\right\rangle_{\left(0, m_{0}-1\right)}+\left\langle\left(J_{2 m_{0}}^{2}-1\right) f f\right\rangle_{\left(m_{0}+1, \infty\right)}
$$

and we have a contradiction.

13 Note from Appendix A that the zeros of $f_{+}(z)$ for $|z|<1$ are simple. 
Appendix B. Analytic properties and bounds. In this appendix we investigate the analytic properties of $f_{+}(z), p(\lambda, n)$ and $p_{+}(z, n)$. We prove that if the coefficients in the recurrence formula satisfy (II.13) and (III.25) then $z f_{+}(z)$ and $p_{+}(z, n)$ are analytic inside the unit circle and continuous on it (see (B.17) and (B.42)). Under the above condition $z f_{+}$and $p_{+}(z, n), z=e^{i \theta}$, are both elements of $A^{+}$(see (B.19) and (B.33)). Bounds on $p(\lambda, n)$ and $\psi(z, n)$ are also derived (see (B.14) and (B.16)).

(A) Properties of $p(\lambda, n)$ and $f_{+}(z)$. Multiplying (II.12) by $z^{n}$ and iterating down yields

$$
\frac{\psi^{*}(z, n)}{\alpha(n)}=1+\sum_{i=0}^{n-1}\left\{\left(1-\frac{a(i+1)^{2}}{a(\infty)^{2}}\right) z^{2}-B(i) z\right\} z^{i} \frac{p(\lambda, i)}{\alpha(i)}
$$

where

$$
\alpha(j)=k(0) \prod_{i=1}^{j} \frac{a(\infty)}{a(i)}, \quad \alpha(0)=k(0)
$$

Now increasing (III.13) by 1 , then multiplying by $z^{n+1}$ and substituting in (B.7) gives

$$
\begin{aligned}
z^{n} p(\lambda, n)= & {\left[1+\sum_{i=0}^{n-1}\left\{\left(1-\frac{a(i+1)^{2}}{a(\infty)^{2}}\right) z^{2}-B(i) z\right\} z^{i} \frac{p(\lambda, i)}{\alpha(i)}-z^{2 n+2}\right.} \\
& \left.\quad-z^{2 n+2} \sum_{i=0}^{n-1}\left\{\left(1-\frac{a(i+1)^{2}}{a(\infty)^{2}}\right) z^{-2}-B(i) z^{-1}\right\} z^{-i} \frac{p(\lambda, i)}{\alpha(i)}\right] \frac{1}{1-z^{2}}
\end{aligned}
$$

which is

$$
\begin{aligned}
= & \frac{1-z^{2 n+2}}{1-z^{2}}+\sum_{i=0}^{n-1}\left(1-\frac{a(i+1)^{2}}{a(\infty)^{2}}\right) z^{2}\left(\frac{1-z^{2 n-2 i-2}}{1-z^{2}}\right) z^{i} \frac{p(\lambda, i)}{\alpha(i)} \\
& -\sum_{i=0}^{n-1} B(i) z\left(\frac{1-z^{2 n-2 i}}{1-z^{2}}\right) \frac{z^{i} p(\lambda, i)}{\alpha(i)} .
\end{aligned}
$$

To get a bound on $z^{n} p(\lambda, n) / \alpha(n)$ we use the technique of successive approximations. Write

$$
\frac{z^{n} p(\lambda, n)}{\alpha(n)}=\sum_{i=0}^{\infty} g(\lambda, n)^{i}
$$

where

$$
g(\lambda, n)^{0}=\frac{1-z^{2 n+2}}{1-z^{2}}
$$

and

$$
\begin{aligned}
g(\lambda, n)^{j}= & \sum_{i=0}^{n-1}\left(1-\frac{a(i+1)^{2}}{a(\infty)^{2}}\right) z^{2}\left(\frac{1-z^{2 n-2 i-2}}{1-z^{2}}\right) g(\lambda, i)^{j-1} \\
& -\sum_{i=0}^{n-1} B(i) z\left(\frac{1-z^{2 n-2 i}}{1-z^{2}}\right) g(\lambda, i)^{j-1}
\end{aligned}
$$


Now there exists a constant $C$ such that

$$
\left|\frac{1-z^{2 n}}{1-z^{2}}\right|<\frac{C n}{1+\left|1-z^{2}\right| n}, \quad n>0,|z|<1 .
$$

Therefore from (B.6),

$$
\left|g(\lambda, n)^{0}\right|<\frac{C(n+1)}{1+\left|1-z^{2}\right|(n+1)} .
$$

Setting $j=1$ in (B.7) and substituting in (B.9) yields

$$
\begin{aligned}
|g(\lambda, n)|^{1}<\sum_{i=0}^{n-1}\left\{\left|1-\frac{a(i+1)^{2}}{a(\infty)^{2}}\right|\left|z^{2}\right|\right. & \frac{C(n-i-1)}{1+\left|1-z^{2}\right|(n-i-1)} \\
& \left.+|B(i)||z| \frac{C(n-i)}{1+\left|1-z^{2}\right|(n-i)}\right\}\left|g^{0}(\lambda, i)\right|
\end{aligned}
$$

which is

$$
<\frac{C_{n}}{1+\left|1-z^{2}\right| n} \sum_{i=0}^{n-1} \frac{C(i+1)}{1+\left|1-z^{2}\right|(i+1)} \gamma(i, z), \quad|z|<1,
$$

where

$$
\gamma(i, z)=\left|1-\frac{a(i+1)^{2}}{a(\infty)^{2}}\right|\left|z^{2}\right|+|B(i)||z| .
$$

Thus by induction

$$
|g(\lambda, n)|^{j}<\frac{C n}{1+\left|1-z^{2}\right| n} \frac{1}{j !}\left[\sum_{i=0}^{n-1} \frac{C(i+1)}{1+\left|1-z^{2}\right|(i+1)} \gamma(i, z)\right]^{j} .
$$

Substituting these results into (B.5) and summing gives

$$
\left|\frac{z_{p}^{n}(\lambda, n)}{\alpha(n)}\right|<\frac{C(n+1)}{1+\left|1-z^{2}\right|(n+1)} \exp \sum_{i=0}^{n-1} \frac{C(i+1)}{1+\left|1-z^{2}\right|(i+1)} \gamma(i, z) \text {. }
$$

It is obvious that

$$
\left\|\frac{z^{n} p(\lambda, n)}{\alpha(n)}\right\|<C(n+1) \exp \sum_{i=0}^{n-1} C(i+1) \gamma(i, 1), \quad z=e^{i \theta} .
$$

Substituting (B.14) into (B.1) yields

$$
\left|\frac{\psi^{*}(z, n)}{\alpha(n)}\right|<1+\sum_{i=0}^{n-1} \frac{C(i+1) \gamma(i, 1)}{1+\left|1-z^{2}\right|(i+1)} \exp \sum_{j=0}^{i-1} \frac{C(j+1)}{1+\left|1-z^{2}\right|(j+1)} \gamma(j, z) .
$$


Now using (III.10) and (B.1) gives

$$
\begin{aligned}
& \left|\frac{C_{+}(z)}{\alpha(\infty)}-\frac{\psi^{*}(z, n)}{\alpha(n)}\right| \\
& \quad<\sum_{i=n}^{\infty} \frac{C(i+1)}{1+\left|1-z^{2}\right|(i+1)} \gamma(i, z) \exp \sum_{j=0}^{i-1} \frac{C(j+1)}{1+\left|1-z^{2}\right|(j+1)} \gamma(j, z) .
\end{aligned}
$$

Therefore if (III.25) is satisfied then $C_{+}(z)$ and, through (III.15), $z f_{+}(z)$ are analytic inside the unit circle and continuous on it. If

$$
\sum_{i=0}^{\infty} \gamma(i, 1)<\infty
$$

then $z f_{+}(z)$ is analytic inside the unit circle and continuous on it except perhaps at $z= \pm 1$. Notice that using (B.15), equations (B.16) and (B.17) are norm inequalities with $|z|=1$ on the L.H.S. and $z=1$ on the R.H.S. of (B.17). Thus

$$
\begin{aligned}
\| \frac{C_{+}\left(e^{i \theta}\right)}{\alpha(\infty)}- & \frac{\psi^{*}\left(e^{i \theta}, n\right)}{\alpha(n)} \| \\
& <\sum_{i=n}^{\infty} C(i+1) \gamma(i, 1) \exp \sum_{j=0}^{i-1} C(j+1) \gamma(j, 1)
\end{aligned}
$$

and we see that $C_{+}\left(e^{i \theta}\right)$, and through (III.15), $e^{i \theta} f_{+}\left(e^{i \theta}\right)$ are elements of $A^{+}$.

(B) Properties of $p_{+}(z, n)$. Let us define

$$
\hat{\Phi}(z, n)=\left(\begin{array}{c}
Q(\lambda, n) \\
\psi_{\alpha}(z, n)
\end{array}\right), \quad n>1,
$$

satisfying (II.17) with initial conditions

$$
Q(\lambda, 1)=\psi_{\alpha}(z, 1)=1 / a(1) k(0) .
$$

Since $\hat{\boldsymbol{\Phi}}$ satisfies (II.17) we define

$$
C_{ \pm \alpha}(z)=W\left[\hat{\Phi}, \Phi_{ \pm}\right]
$$

which in component form is

$$
C_{+\alpha}(z)=p_{+}(z, n) \psi_{\alpha}(z, n)-p(\lambda, n) \psi_{+}(z, n)
$$

and

$$
C_{-\alpha}(z)=p_{-}(z, n) \psi_{\alpha}(z, n)-p(\lambda, n) \psi_{-}(z, n) .
$$

Letting $n \rightarrow \infty$ in (B.23) using (III.4) yields

$$
C_{+\alpha}(z)=\lim _{n \rightarrow \infty} z^{n} \psi_{\alpha}(z, n) .
$$


Since $\psi_{\alpha}(z, n)$ and $Q(\lambda, n)$ satisfy (III.13) we can write

$$
\begin{aligned}
\frac{z^{n} Q(\lambda, n)}{\alpha(n)}= & \frac{z\left(1-z^{2 n}\right)}{a(\infty) k(0)^{2}\left(1-z^{2}\right)} \\
& +\sum_{i=1}^{n-1}\left\{1-\frac{a(i+1)^{2}}{a(\infty)^{2}}\right\} z^{2}\left(\frac{1-z^{2 n-2 i-2}}{1-z^{2}}\right) z^{i} Q(\lambda, i) \\
& -\sum_{i=1}^{n-1} B(i) z\left(\frac{1-z^{2 n-2 i}}{1-z^{2}}\right) z^{i} Q(\lambda, i) .
\end{aligned}
$$

Applying the procedures that led to (B.14), (B.15), and (B.17) yields

$$
\begin{aligned}
\left|\frac{z^{n} Q(\lambda, n)}{\alpha(n)}\right| \leqslant & \frac{C n}{a(\infty) k(0)^{2}\left(1+\left|1-z^{2}\right| n\right)} \\
& \cdot \exp \left[\sum_{i=1}^{n-1} \frac{C i \gamma(i, z)}{a(\infty) k(0)^{2}\left(1+\left|1-z^{2}\right| i\right)}\right], \\
\left|\frac{\psi_{\alpha}^{*}(z, n)}{\alpha(n)}\right| \leqslant & \left(\frac{|z|}{a(\infty) k(0)^{2}}\right) \sum_{i=1}^{n-1} \frac{C i \gamma(i, z)}{a(\infty) k(0)^{2}\left(1+\left|1-z^{2}\right| i\right)} \\
& \cdot \exp \sum_{j=1}^{i-1} \frac{C j \gamma(j, z)}{a(\infty) k(0)^{2}\left(1+\left|1-z^{2}\right| j\right)},
\end{aligned}
$$

and

$$
\begin{aligned}
\left|\frac{C_{+\alpha}(z)}{\alpha(\infty)}-\frac{\psi_{\alpha}^{*}(z, n)}{\alpha(n)}\right| \leqslant & \sum_{i=n}^{\infty} \frac{C i \gamma(i, z)}{a(\infty) k(0)^{2}\left(1+\left|1-z^{2}\right| i\right)} \\
& \cdot \exp \sum_{j=1}^{i-1} \frac{C j \gamma(j, z)}{a(\infty) k(0)^{2}\left(1+\left|1-z^{2}\right| j\right)}
\end{aligned}
$$

Therefore $C_{+\alpha}(z)$ has analytic properties similar to those of $C_{+}(z)$. Setting $|z|=1$ on the L.H.S. and $z=1$ on the R.H.S. makes (B.27), (B.28) and (B.29) norm inequalities thus making $C_{+\alpha}(z)$ an element of $A^{+}(z)$.

Multiplying (B.23) by $Q(\lambda, n)$ and (III.8) by $p(\lambda, n)$ then subtracting one from the other yields

$$
\begin{aligned}
\left(C_{+}(z) Q(\lambda, n)-\right. & \left.C_{+\alpha}(z) p(\lambda, n)\right) \\
& =p_{+}(z, n)\left[\psi(z, n) Q(\lambda, n)-\psi_{\alpha}(z, n) p(\lambda, n)\right]
\end{aligned}
$$

Now

$$
\psi(z, n) Q(\lambda, n)-\psi_{\alpha}(z, n) p(\lambda, n)=W[\Phi, \hat{\Phi}]
$$

which is

$$
=\left[\psi(z, 1) Q(\lambda, 1)-\psi_{\alpha}(z, 1) p(\lambda, 1)\right]=-z / a(\infty) .
$$

Thus (B.30) becomes

$$
p_{+}(z, n)=\frac{a(\infty)}{z}\left[C_{+\alpha}(z) p(\lambda, n)-C_{+}(z) Q(\lambda, n)\right], \quad n>1,|z|=1 .
$$


Using similar procedures the following equation for $\psi_{+}(z, n)$ can be derived

$$
\psi_{+}(z, n)=\frac{a(\infty)}{z}\left[C_{+}(z) \psi_{\alpha}(z, n)-C_{+\alpha}(z) \psi(z, n)\right], \quad n \geqslant 1 .
$$

Letting $n \rightarrow \infty$ in (B.1) using (III.10) and (B.25) gives

$$
\frac{C_{+}(z)}{\alpha(\infty)}=\frac{\psi^{*}(z, n)}{\alpha(n)}+\sum_{i=n}^{\infty}\left\{\left(1-\frac{a(i+1)^{2}}{a(\infty)^{2}}\right) z^{2}-B(i) z\right\} z^{i} \frac{p(\lambda, i)}{\alpha(i)}
$$

and

$$
\frac{C_{+\alpha}(z)}{\alpha(\infty)}=\frac{\psi_{\alpha}^{*}(z, n)}{\alpha(n)}+\sum_{i=n}^{\infty}\left\{\left(1-\frac{a(i+1)^{2}}{a(\infty)^{2}}\right) z^{2}-B(i) z\right\} z^{i} \frac{Q(\lambda, i)}{\alpha(i)} .
$$

Substituting these equations into (B.33) yields

$$
\begin{aligned}
p_{+}(z, n)= & z^{n} \frac{a(\infty)}{z} \frac{\alpha(\infty)}{\alpha(n)}\left[\psi_{\alpha}(z, n) p(\lambda, n)-\psi(z, n) p(\lambda, n)\right] \\
& +\frac{a(\infty) \alpha(\infty)}{z} \sum_{i=n+1}^{\infty}\left\{\left(1-\frac{a(i+1)^{2}}{a(\infty)^{2}}\right) z^{2}-B(i) z\right\} \frac{z^{i}}{\alpha(i)} \\
& \cdot[Q(\lambda, i) p(\lambda, n)-p(\lambda, i) Q(\lambda, n)]
\end{aligned}
$$

which is using (B.32)

$$
\begin{aligned}
= & z^{n} \prod_{i=n+1}^{\infty} \frac{a(\infty)}{a(i)}+\frac{a(\infty) \alpha(\infty)}{z} \sum_{i=n+1}^{\infty}\left\{\left(1-\frac{a(i+1)^{2}}{a(\infty)^{2}}\right) z^{2}-B(i) z\right\} \\
& \cdot \frac{z^{i}}{\alpha(i)}[Q(\lambda, i) p(\lambda, n)-p(\lambda, i) Q(\lambda, n)] .
\end{aligned}
$$

Notice that

$$
g(\lambda, i, n)=(Q(\lambda, i) p(\lambda, n)-p(\lambda, i) Q(\lambda, n))
$$

and

$$
\hat{g}(z, i, n)=\left[\psi_{\alpha}(z, i) p(\lambda, n)-\psi(z, i) Q(\lambda, n)\right]
$$

satisfy (II.17) with initial conditions

$$
g(\lambda, n+1, n)=\hat{g}(z, n+1, n)=1 / a(n+1) .
$$

Therefore $z^{i-n-1} g(\lambda, i, n)$ and $z^{i-n-1} \hat{g}(z, i, n)$ are polynomials in $z$ of degree $2(i-n-1)$. Since $g(\lambda, i, n)$ and $\hat{g}(z, i, n)$ satisfy (III.13) we can use the procedures leading to (B.4) to derive the following formula

$$
\begin{aligned}
z^{i-n-1} \frac{g(\lambda, i, n)}{\alpha(i)}= & \frac{1}{a(n+1) \alpha(i)}\left[\frac{1-z^{2(i-n)}}{1-z^{2}}\right] \\
& +\sum_{j=n+1}^{i-1}\left(1-\frac{a(j+1)^{2}}{a(\infty)^{2}}\right) z^{2}\left(\frac{1-z^{2(i-j-1)}}{1-z^{2}}\right) z^{j-n-1} \frac{g(\lambda, j, n)}{\alpha(j)} \\
& -\sum_{j=n+1}^{i-1} B(j) z\left(\frac{1-z^{2(i-j)}}{1-z^{2}}\right) z^{j-n-1} \frac{g(\lambda, j, n)}{\alpha(j)}
\end{aligned}
$$


Applying the same procedures that led to (B.14) we find

$$
\begin{aligned}
\left|z^{i-n-1} \frac{g(\lambda, i, n)}{\alpha(i)}\right|< & \frac{1}{a(n+1) \alpha(i)} \frac{C(i-n)}{1+\left|1-z^{2}\right|(i-n)} \\
& \cdot \exp \sum_{j=n+1}^{i-1} \frac{C}{a(n+1) \alpha(j)} \frac{(j-n)}{1+\left|1-z^{2}\right|(j-n)} \gamma(j, z) .
\end{aligned}
$$

Substituting (B.41) into (B.37) yields

$$
\begin{aligned}
\left|p_{+}(z, n)\right|<\left|z^{n} \prod_{i=n+1}^{\infty} \frac{a(\infty)}{a(i)}\right| & \\
& +\frac{a(\infty)}{a(n+1)}\left|z^{n+1}\right| \sum_{i=n+1}^{\infty} \frac{\alpha(\infty)}{\alpha(i)}\left\{\left|1-\frac{a(i+1)^{2}}{a(\infty)^{2}}\right||z|+|B(i)|\right\} \\
& \cdot \frac{C(i-n)}{1+\left|1-z^{2}\right|(i-n)} \exp \sum_{j=n+1}^{i-1} \frac{C(j-n) \gamma(j, z)}{a(n+1) \alpha(j)\left(1+\left|1-z^{2}\right|(j-n)\right)}
\end{aligned}
$$

Since the right-hand side of (B.33) and (B.34) satisfy (II.7) with boundary conditions (III.4) $p_{+}(z, n)$ has analytic properties similar to those of $C_{+}(z)$ and $C_{+\alpha}(z)$ and is also an element of $A^{+}$. It is easy to see that (B.42) becomes a norm inequality if one follows the procedures leading to (B.19).

Once having found $d \rho(\lambda)$ it is clear that

$$
Q(\lambda, n)=\int_{a}^{b} \frac{\left(p(\lambda, n)-p\left(\lambda^{\prime}, n\right)\right)}{\lambda-\lambda^{\prime}} d \rho\left(\lambda^{\prime}\right), \quad n>1,
$$

and

$$
\begin{aligned}
\psi_{\alpha}(z, n)= & \int_{a}^{b} \frac{\psi(z, n)-\psi\left(z^{\prime}, n\right)}{\lambda-\lambda^{\prime}} d \rho\left(\lambda^{\prime}\right) \\
& +\frac{a(n)}{a(\infty)} \int_{a}^{b}\left(z-z^{\prime}\right) \frac{p\left(\lambda^{\prime}, n-1\right)}{\lambda-\lambda^{\prime}} d \rho\left(\lambda^{\prime}\right), \quad n>1 .
\end{aligned}
$$

Since the boundary conditions (B.21) are equivalent to

$$
Q(\lambda, 0)=0, \quad Q(\lambda, 1)=1 / a(1) k(0),
$$

we can apply the steps that led to equation (III.20). Thus

$$
\begin{aligned}
f_{+\alpha}(z) & =\frac{a(\infty)}{k(0) z} C_{+\alpha}(z) \\
& =\frac{a(n+1)}{k(0)}\left[Q(\lambda, n+1) p_{+}(z, n)-p_{+}(z, n+1) Q(\lambda, n)\right], \quad n>0,
\end{aligned}
$$

which is

$$
=1 / k(0)^{2} p_{+}(z, 0)
$$


Substituting (V.3) into (B.42) yields the following bound

$$
\begin{aligned}
\sum_{m=n+1}^{\infty}|\alpha(n, m)| \leqslant & \frac{a(\infty)}{a(i+1)} \sum_{i=n+1}^{\infty} \gamma(i, 1) C(i-n) \\
& \cdot \exp \sum_{j=n+1}^{\infty} \frac{C(j-n) \gamma(j, 1)}{a(n+1) \alpha(i)}
\end{aligned}
$$

where $\alpha(n, m)$ is defined in (V.12). From (V.10)

$$
|\omega(n, m)|<|\alpha(n, m)|+\sum_{n^{\prime}=n+1}^{\infty}\left|\alpha\left(n, n^{\prime}\right)\right|\left|\omega\left(n^{\prime}, m\right)\right| \text {. }
$$

Summing on $m$ from $l$ to $\infty, l>n+1$

$$
\sum_{m=l}^{\infty}|\omega(n, m)| \leqslant \sum_{m=l}^{\infty}|\alpha(n, m)|+\sum_{m=l} \sum_{n^{\prime}=n+1}^{\infty}\left|\alpha\left(n, n^{\prime}\right)\right|\left|\omega\left(n^{\prime}, m\right)\right| .
$$

From (B.45) there exists an $N$ such that

$$
\sum_{n^{\prime}=n+1}^{\infty}\left|\alpha\left(n, n^{\prime}\right)\right|<1
$$

for all $n>N$. From (V.13) $\omega(n, m)=\omega(n+m)$. Thus (B.50) can be rewritten as

$$
\sum_{m=l+n}^{\infty}|\omega(m)| \leqslant \sum_{m=l}^{\infty}|\alpha(n, m)|+\sum_{m=l+n^{\prime}}|\omega(m)| \sum_{n^{\prime}=n+1}^{\infty}\left|\alpha\left(n, n^{\prime}\right)\right|
$$

which is

$$
\leqslant \sum_{m=l}^{\infty}|\alpha(n, m)|+\sum_{m=l+n}^{\infty}|\omega(m)| \sum_{n^{\prime}=n+1}^{\infty}\left|\alpha\left(n, n^{\prime}\right)\right| .
$$

Combining the second term on the right-side with the left-hand side yields

$$
\sum_{m=l+n}^{\infty}|\omega(m)| \leqslant \frac{\sum_{m=l}^{\infty}|\alpha(n, m)|}{1-\sum_{m=n+1}^{\infty}|\alpha(n, m)|} .
$$

From (V.16), $\omega(m)$ is bounded for all $m$ and therefore summable. These techniques can be applied to (V.14) with similar results.

\section{REFERENCES}

1. Z. S. Agranovich and V. A. Marchenko, The inverse problem of scattering theory, translated from the Russian by B. D. Seckler, Gordon and Breach, New York, 1963.

2. N. I. Akhiezer, The classical moment problem and some related questions in analysis, translated from the Russian by N. Kemmer, Hafner, New York, 1965.

3. G. Baxter, A convergence equivalence related to polynomials orthogonal on the unit circle, Trans. Amer. Math. Soc. 99 (1961), 471-487.

4. K. M. Case, Orthogonal polynomials from the viewpoint of scattering theory, J. Math. Phys. 15 (1974), 2166-2174.

5. K. M. Case and S. C. Chui, The discrete version of the Marchenko equation in the inverse scatering problem, J. Math. Phys. 14 (1973), 1643-1650.

6. J. S. Geronimo, Scattering theory and orthogonal polynomials, Doctoral Dissertation, Rockefeller Univ., 1977.

7. J. S. Geronimo and K. M. Case, Scattering theory and polynomials orthogonal on the unit circle, J. Math. Phys. 201 (1979), 299. 
8. G. S. Guseinov, The determination of an infinite Jacobi matrix from the scattering data, Soviet Math. Dokl. 17 (1976), 596-600.

9. Paul Nevai, Orthogonal polynomials, Mem. Amer. Math. Soc., no. 18, 1979, 185 pp.

10. G. Szegö, Orthogonal polynomials, Amer. Math. Soc. Colloq. Publ., vol. 23, Amer. Math. Soc., Providence, R. I., 1939, p. 27; 4th edition, 1975.

11. A. Zygmund, Trigonometric series, vol. 1, 2nd ed., Cambridge Univ. Press, New York, 1959, pp. 245-246.

School of Mathematics, Georgin Institute of Technology, Atlanta, Georgin 30332

Department of Physics, Rockefeller University, New York, New York 10021 